\title{
Geologia
}

\section{Aplicação da abordagem de Importação Semântica (IS) para caracterização de contatos litológicos em modelos de inferência espacial}

\author{
Fábio Roque da Silva Moreira \\ Geólogo (USP) - Mestre em Sensoriamento Remoto (INPE). Instituto Nacional de Pesquisas Espaciais - INPE, \\ C.P. 515, São José dos Campos - SP.E-mail: fmoreira@ltid.inpe.br \\ Raimundo de Almeida Filho \\ Geólogo (UnB) - Doutor em Geologia Geral Aplicada (USP). Instituto Nacional de Pesquisas Espaciais - INPE, \\ C.P. 515, São José dos Campos - SP.E-mail: rai@ltid.inpe.br
}

Gilberto Câmara

Eng. Eletrônico (ITA) - Doutor em Computação (INPE). Instituto Nacional de Pesquisas Espaciais - INPE, C. P. 515, São José dos Campos - SPE-mail: gillberto@dpi.inpe.br.

\section{Resumo}

O presente trabalho descreve o uso da abordagem da importação semântica (IS) como modo de caracterização do tipo de contato geológico em processos de análise espacial. A técnica utiliza lógica fuzzy na representação da característica espacial de contatos litológicos (definidos e inferidos) em um modelo de inferência espacial para mapeamento de áreas potenciais para a prospecção de minerais radioativos, no maciço alcalino de Poços de Caldas. O procedimento permitiu utilizar mais eficientemente unidades litológicas como critério diagnóstico num modelo prospectivo desenvolvido em SIG. Funções lineares fuzzy foram aplicadas para criar superfícies contínuas que expressam com mais confiança a distribuição espacial de cada unidades litológica. O mapa litológico resultante foi integrado a outras evidências (e.g. intensidade gama-radiométrica e feições estruturais) através de uma soma ponderada. $\mathrm{O}$ resultado final foi arbitrariamente fatiado em quatro categorias de potencialidade (nula, baixa, média e alta). O cenário fuzzy foi avaliado qualitativamente e quantitativamente comparando seus resultados com os obtidos por Almeida-Filho (1995), que utilizou os modelos booleano e soma ponderada. A comparação dos diferentes modelamentos demostrou que a performance do cenário fuzzy foi ligeiramente melhor. Uma área de $30,43 \mathrm{~km}^{2}$ (4,18\% do complexo alcalino) encerrou 27 ocorrências minerais, enquanto cada prévio modelo encerrou apenas 24 , em áreas aproximadamente similares. Foram também avaliadas a correlação entre as ocorrências minerais e as classes de potencialidade dos diferentes cenários. As classes "alto" e "médio potencial" do cenário fuzzy obtiveram probabilidade a posteriore 12,9 e 5,7 vezes maior do que a probabilidade a priori. Esses valores foram, respectivamente, 12,6 e 4,97 para as mesmas classes do método soma ponderada. O modelo booleano teve 5,78 para a classe favorável. Esses resultados demostraram que a teoria da lógica fuzzy permite um melhor refinamento no modelamento dos dados, melhorando a habilidade de representá-los espacialmente em superfícies contínuas.

\section{Abstract}

This work discusses the use of semantic import approach (SI) as a way to characterize geological boundaries in spatial analysis procedures. The technique applies fuzzy sets to represent the nature of the lithological boundaries (defined and inferred) in a spatial inference model used to map potential areas for radioactive mineral prospecting in the Poços de Caldas alkaline complex. The procedure permits use of more efficient lithological units as diagnostic criteria in a prospecting model developed in GIS. Linear fuzzy functions were applied to create continuous surfaces that express more faithfully the spatial distribution of each lithological unit. The final lithological map was integrated with other field evidences (e.g. gamma-ray total counts and structural features) through a weighted means sum. The resulting product was arbitrarily sliced in four potential categories (null, low, medium and high). The fuzzy scenario was qualitatively and quantitatively compared with previous results obtained by Almeida-Filho (1995). This author used boolean algebra and weighted means based models to map potential areas. The comparison of both procedures showed that the fuzzy scenario was slightly better. A target area of $30,43 \mathrm{Km}^{2}$ (4,18\% of the alkaline complex) included 27 mineral occurrences, whereas each previous model encompassed only 24 in an approximately similar area. We also assessed the correlation of mineral occurrences in relation to the high and medium potential classes for the different scenarios. The high and medium potential classes of the fuzzy scenario showed posterior probabilities 12.9 and 5.7 higher than the prior probabilities. These values were, respectively, 12.6 and 4.97 for the same classes defined by Almeida Filho (1995). For the potential class of the Boolean model this value was 5.78 . These results show that fuzzy set theory enables a better refinement of the data modeling, improving the ability to spatially represent geological boundaries as continuous surfaces.

Keywords: semantic import approach for boundaries, fuzzy sets, spatial analysis, GIS.

Artigo recebido em 15/07/2002 e aprovado em 01/10/2002. 


\section{Introdução}

Em mapas geológicos contatos litológicos em geral definem limites estanques entre diferentes unidades. Embora eficiente do ponto de vista prático, esse tipo de representação cartográfica é, muita vezes, simplista do ponto de vista geológico, pois pode suprir informação sobre a real natureza das mudanças verificadas em campo. Tais casos podem constituir uma limitação quando essas unidades são utilizadas como elementos de entradas em modelos empíricos de prospeção, desenvolvido com o emprego de Sistemas de Informações Georreferenciadas (SIG's).

É sabido que variações litológicas podem ocorrer de forma abrupta ou gradual. Um dique intrusivo ou uma falha podem colocar em contato abrupto unidades distintas do ponto de vista geológico, mas nem sempre acontece dessa forma. Na natureza, a maioria das mudanças é feita de modo gradual, através de zonas de transição. Como essas transições são, em geral, difíceis de serem representadas em mapa, convencionase, mais ou menos arbitrariamente, uma linha divisória entre áreas com certas características predominantes. Entretanto, em modelos de inferência espacial com emprego de Sistemas de Informações Georrefenciadas (SIG's), esse tipo de representação de contatos pode levar a interpretações equivocadas sobre a informação espacial do atributo (Wang \& Brent Hall, 1996). Sobre essa questão, Burrough e Heuvelink (1992) demonstraram, através de modelo de inferência espacial, como a utilização de contatos rígidos na representação espacial de dados litológicos contínuos favorece a propagação de erros que podem levar a cenários pouco realistas.

De modo a utilizar mais eficazmente unidades litológicas como critérios diagnósticos em modelos prospectivos assistidos por computador, Burrough e McDonnell (1998) desenvolveram a abordagem denominada "Importação Semântica para Contatos" (IS). Ela utiliza conjuntos fuzzy (funções fuzzy) na caracterização espacial dos contatos, possibilitando a caracterização, tanto da informação do tipo de contato (abrupto, gradual, etc.) quanto da incerteza de sua localização.
O presente artigo exemplifica o emprego da abordagem IS, na representação de contatos (definido, inferido) de unidades litológicas, adotadas em um modelo prospectivo. Como exemplo utilizou-se um modelo prospectivo empírico previamente definido por AlmeidaFilho (1995), visando à definição de áreas potenciais para a ocorrência de minerais radioativos no complexo alcalino de Poços de Caldas. Utilizando abordagem IS, unidades litológicas consideradas como hospedeiras potenciais dessas mineralizações foram combinadas com outras evidências (anomalia gama-radiométrica e dados estruturais) em um processo de inferência espacial definido pela teoria dos conjuntos fuzzy. Os resultados foram comparados àqueles obtidos por Almeida-Filho (1995), que utilizou técnicas mais simples de álgebra booleanas e cruzamento ponderado dos diferentes critérios.

\section{2. Área de estudo}

A área de estudo engloba o platô de Poços de Caldas, localizado na divisa dos Estados de Minas Gerais e São Paulo, a aproximadamente $300 \mathrm{~km}$ da capital paulista (Figura 1a). O maciço, de formato grosseiramente circular, possui área de cerca de $750 \mathrm{~km}^{2}$, com diâmetro de aproximadamente $35 \mathrm{~km}$.

Rochas de filiação alcalina, com predominância de nefelinas-sienitos (tinguaítos, fonólitos, foiaítos), predominam no complexo de Poços de Caldas, um corpo intrusivo de idade mesozóica-cenozóica (Figura 1b). Os diversos tipos litológicos de origem alcalina podem ser reunidos em três grupos principais: material vulcânico; rochas efusivas e hipabissais; e rochas plutônicas (Fraenkel et al., 1985). Material vulcânico, representado por brechas, tufos e aglomerados, aflora na porção noroeste do maciço. Rochas efusivas e hipabissais são representadas por fonólitos e tinguaítos, respectivamente, com ampla distribuição por todo o maciço. Rochas plutônicas, constituídas por foiaítos, lujaritos e, em menor proporção, chibinitos, ocorrem encaixadas nas fáceis efusivas e hipabissais. Outra litologia importante no maciço é resultante da alteração hidrotermal e intempérica dos tinguaítos, denominada de "rocha potássica", um dos mais importantes controles litológicos das mineralizações radioativas.

Dados geocronológicos K-Ar (Bushee, 1970) permitem estimar a duração da evolução magmática do complexo alcalino de Poços de Caldas em cerca de 30 m.a., tendo as primeiras manifestações ocorrido no Cretáceo Superior (87m.a.). Estudos realizados por Ellert (1959) permitiram-lhe reconhecer a existência de 6 fases na formação do complexo alcalino: (a) soerguimento do embasamento; (b) atividades vulcânicas; (c) formação da caldeira; (d) atividade magmática alcalina; (e) formação dos diques anelares; (f) intrusões de foiaítos, chibinitos e lujaritos.

Dois grandes sistemas de falhas com direções predominantes N60W e N40E encontram-se presentes no complexo alcalino. O primeiro está relacionado com a tectônica regional e o segundo, com o processo formador da caldeira (Fraenkel et al., 1985). Almeida-Filho e Paradella (1977), através da interpretação de imagens do multispectral scanner (MSS) do satélite Landsat-1, indicam a existência de 7 estruturas circulares no interior da caldeira de Poços de Caldas, possivelmente associadas à presença de cones vulcânicos (Figura 1c). A presença de várias ocorrências minerais radioativas ao longo das bordas dessas estruturas, levou esses autores a considerarem que essas feições constituíram um controle estrutural regional das mineralizações.

São conhecidas 48 ocorrências de mineralizações radioativas (Figura 1c), que podem ser agrupadas em três associações: urânio-zircônio, tório-terras raras e urânio-molibdênio (Tolbert, 1966; Oliveira, 1974, Fraenkel et al.,1985). A associação urânio-zircônio é a mais comum, ocorrendo como depósitos aluviais, eluviais e na forma de veios e lentes. A associação tório-terras raras representa o segundo tipo, sendo o depósito de Morro de Ferro o melhor representante dessa associação. A associação urâniomolibdênio é a mais importante, ocorrendo predominantemente em faixas filoneanas ou como corpos lenticulares. 


\section{Modelo prospectivo}

Modelos de integração e inferência espacial desenvolvidos em SIG baseiam-se principalmente em relações empíricas definidas com a ajuda descriti- va do "modelo de depósito". O poder explicativo desses modelos depende fundamentalmente do conhecimento geológico prévio sobre a área de estudo, que permitirá a seleção dos critérios di- agnósticos mais apropriadas para a "alimentação" do modelo.

A complexidade dos fenômenos envolvidos no processo formador do complexo alcalino e de suas mineraliza-
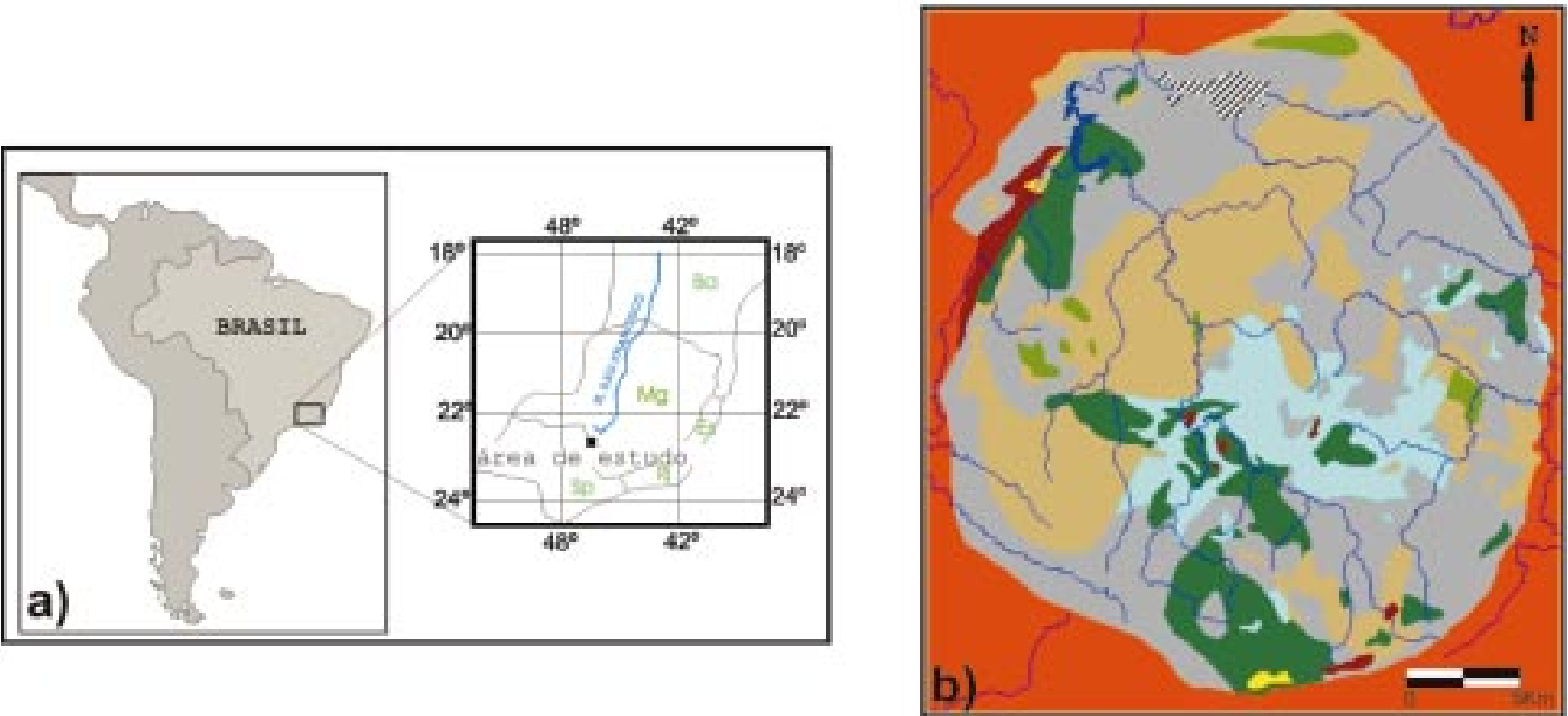

Legenda

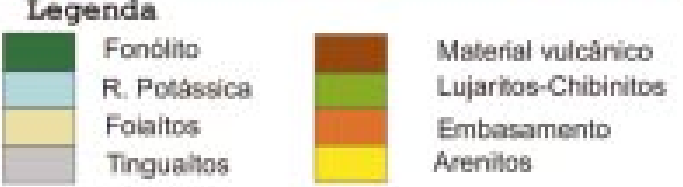

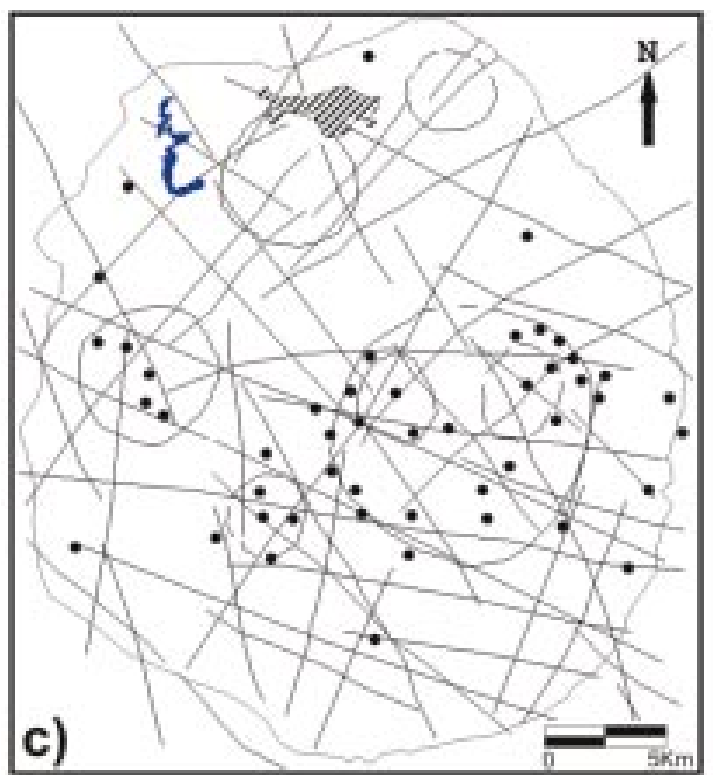

Legenda

Estr. Circulares

Lineamentos

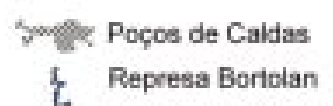

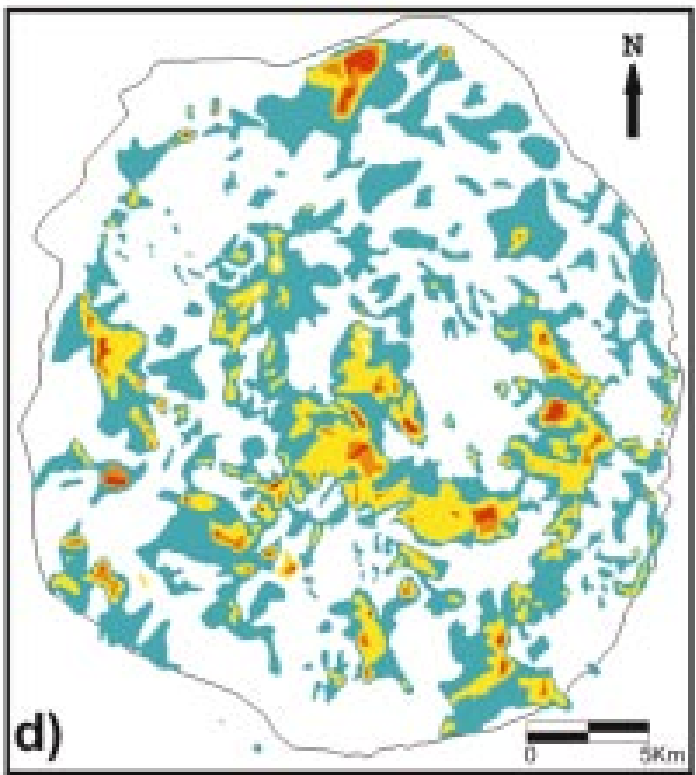

Legenda

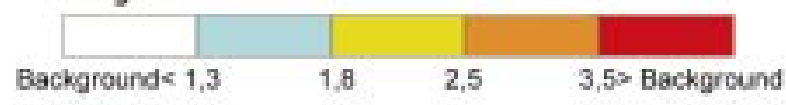

Figura 1 - Localização da área de estudo (a); mapa litológico do maciço alcalino (Fonte: NUCLEBRÁS, 1975a) (b); mapa de lineamentos estruturais (Modificado: Almeida-Filho, 1995) (c); e mapa de anomalias intensidade gama-radiometria (Fonte: NUCLEBRÁS, 1975b) (d). 
ções torna muito difícil a concepção de um modelo prospectivo empírico que contemple todas as nuanças envolvidas no processo mineralizador. Este envolve aspectos tectônicos, estruturais, litológicos e intempéricos, que apresentam particularidades de uma área para outra. A despeito dessas dificuldades, Almeida-Filho (1995) identificou certas características comuns às ocorrências minerais no maciço alcalino, as quais foram assumidas como parâmetros controladores dessas ocorrências, constituindo critérios diagnósticos para a pesquisa de minerais radioativos na área:

- Litologias favoráveis: presença de controles litológicos, representados por rochas potássicas, material vulcânico e corpos intrusivos de foiaítos.

- Falhas/fraturas: presença de falhas/ fraturas, condicionando o alojamento de veios e lentes mineralizados.

- Estruturas circulares: presença de cones vulcânicos no interior da cratera, condicionando a ocorrência de mineralizações radioativas em suas bordas.

- Gama-radiometria: ocorrência de valores anômalos de radioatividade total, indicativos da presença de minerais radioativos (Figura $1 \mathrm{~d}$ ).

\section{Banco de Dados Georreferenciados}

Para a realização do estudo foi montado um banco de dados espacial no SPRING (Sistema de Processamento de Informações Georreferenciadas), desenvolvido pelo Instituto Nacional de Pesquisas Espaciais (Câmara et al., 1996). Esses dados foram convertidos para a projeção UTM/SAD69, utilizando como referência cartográfica as folhas de Poços de Caldas, Santa Rita de Caldas e Andrada, na escala 1:50.000, produzidas pela Fundação Instituto Brasileiro de Geografia e Estatística - IBGE.

\section{Metodologia}

A teoria dos conjuntos fuzzy tem sido amplamente utilizada em trabalhos de inferência espacial desenvolvidos em SIG's (Burrough, 1989; Burrough \& Heu- velink, 1992; Banai, 1993; Altman,1994). As vantagens do modelamento fuzzy são inúmeras, quando comparadas aos modelamentos convencionais que forçam os especialistas a definirem regras dicotômicas rígidas, que diminuem a habilidade de articular eficientemente soluções para problemas complexos, tão comuns em processos naturais.

Entretanto essa visão dicotômica (booleana) é assumida pela maioria dos SIG's, admitindo-se que fenômenos naturais podem ser modelados por objetos discretos com atributos precisos, tais como pontos, linhas e polígonos. Como mostrado por Altman (1994), essa representação espacial rígida de informações naturais geralmente introduz erros que se propagam desde o início até o final dos processos de inferência espacial assistidos por computador.

Os sistemas de inferências baseados em lógica fuzzy, por outro lado, possibilitam a captura do conhecimento de maneira mais similar àquela adotada em "modelos cognitivos". Nessa concepção de modelamento de sistemas complexos, os mecanismos envolvidos são representados através de variáveis lingüísticas que permitem lidar de modo mais adequado com dados imprecisos, incompletos, ambíguos, e/ou vagos, tão comuns em sistemas geológicos (Fang, 1996). Um exemplo são estudos de grãos em sedimentologia. Quando o geólogo deseja definir o tipo de transporte e/ou maturidade de sedimentos a partir do padrão de arredondamento de grãos, vale-se de um modelo cognitivo, definindo o padrão de arredondamento como uma variável que pode assumir valores lingüísticos (arredondado, subarredondado, sub-anguloso, anguloso) ao invés de valores quantitativos.

Para a realização da inferência fuzzy, os critérios diagnósticos (litologias, anomalias gama-radiométricas, feições estruturais) foram representados em forma numérica, tipo modelo numérico de terreno (MNT). Para isso, os mapas temáticos foram ponderados para valores entre 0 e 1 (membros fuzzy). Essa representação pretendeu expressar, de modo semelhante ao modelo cognitivo humano, o grau de aceitação da evidên- cia para com o modelo prospectivo adotado.

$\mathrm{Na}$ atribuição dos membros fuzzy litologia, o objetivo foi gerar um plano de informação que expressasse o grau de aceitação de cada unidade litológica no modelo prospectivo, considerando também a informação semântica dos tipos de contato (definido ou inferido). Para isso, a primeira etapa foi a construção de um plano de informação (PI), com representação temática, com 2 classes de máscaras que diferenciavam os corpos litológicos com contatos definidos daqueles com contatos inferidos. Este PI serviu de "condicionante espacial" para a aplicação das diferentes funções membro fuzzy. A seguir o PI litologia foi desmembrado em suas diferentes unidades litológicas, de modo que cada unidade fosse confrontada com as demais. Em teoria de conjuntos seria o conjunto A confrontado com o não-A $(\bar{A}=T-A)$. Em seguida foram criados mapas de distâncias, a partir dos contatos das unidades litológicas. Esses mapas serviram de base numérica para a aplicação das funções membro fuzzy, que devem mapear a informação dos tipos de contatos.

O passo seguinte constou da construção dos PI's com os membros fuzzy que expressassem o grau de aceitação de cada unidade litológica, considerando o tipo de contato. Eles foram obtidos através da aplicação das funções membrofuzzy sobre os mapas de distância de cada unidade litológica, controladas pelas máscaras e por um corredor de $100 \mathrm{~m}$ de largura ao longo dos contatos inferidos. O corredor equivale a uma zona de transição e serve para delimitar a região onde os membros fuzzy expressam a possibilidade da localização dos contatos inferidos. A formulação apresentada a seguir exemplifica a aplicação das diferentes funções membro fuzzy controladas pelos condicionantes espaciais para a rocha potássica:

$M F_{\text {R.potássica }}=0$ Se Classe. Máscara $=$ contato definido E Classe.PI binário = Nãorocha potássica

$M F_{\text {R.potássica }}=1$ Se Classe. Máscara $=$ contato definido $\mathbf{E}$ Classe.PI binário = rocha potássica 
$M F_{\text {R.potássica }}=(0.005 *$ dist $)+0.5$ Se Classe.Máscara $=$ contato inferido $\mathbf{E}$ Classe.PI binário = rocha potássica $\mathrm{E}$ distância $\leq 100 \mathrm{~m}$;

$M F_{\text {R.potássica }}=(-0.005 * \mathrm{dist})+0.5 \mathrm{Se}$ Classe. Máscara $=$ contato inferido $\mathbf{E}$

Classe.PI binário = Não-R.potássica $\mathrm{E}$ distância $\leq 100 \mathrm{~m}$.

As operações apresentadas anteriormente garantem que a localização do contato rígido original coincida com o ponto de cruzamento $\mathrm{MF}=0,5$. Pontos sobre as unidades litológicas e na zona de transição assumem valores entre $1 \mathrm{e}$ 0,5 . Fora do contato, mas dentro da zona de transição, recebem valores menores que 0,5 , conforme o distanciamento do contato.

A Figura 2 ilustra o resultado do procedimento aplicado em um trecho do contato entre as unidades litológicas "rocha potássica" e "material vulcânico", separadas pelo contato rígido original. $\mathrm{Na}$ parte esquerda da figura, estão indicados os membros fuzzy referentes à unidade litológica "rocha potássica" e, na parte direita, estão os membros fuzzy para a unidade litológica "material vulcânico". As linhas pontilhadas demarcam a zona de transição de $100 \mathrm{~m}$ entre as duas unidades. As funções membro fuzzy que graduam os elementos são mostradas no topo da figura.

Os PI's litológicos fuzzy foram combinados através de uma soma ponderada (Equação 5). Os pesos, escolhidos empiricamente, pretenderam expressar o grau de importância de cada unidade litológica para com o modelo prospectivo adotado. Eles foram obtidos através da aplicação de uma função linear $(f(x)=$ 0,0167.x) sobre os pesos definidos por Almeida-Filho (1995).

$M F_{\text {litógia }}=R \cdot$ potássica + Lujarito $/$ chibinito $+(0.5 *$ Foiaíto $)+(0.33 *$ Mat. vulcânico)

O PI litológico fuzzy total foi finalmente integrado às demais evidências (anomalias gama-radiométricas, feições estruturais), através de uma análise multicritério definida pelo operador fuzzy ponderado. Os pesos de integração foram definidos pela técnica AHP (Analytic Hierarchy Process) (Saaty, 1992), que permite a definição de pesos que expressam, de modo hierárquico, os membros fuzzy, através de uma comparação feita dois a dois entre as evidências.

Nesse processo, a comparação em pares funciona como uma análise decomposta, que finaliza com uma síntese de recomposição, pela agregação dos valores membro dos elementos, em um método de avaliação unificado (Banai, 1993). O resultado final é um PI numérico que expressa, em cada ponto da grade, a potencialidade da ocorrência de minerais radioativos. Esse PI foi fatiado em intervalos arbitrários de potencialidade (nula, baixa, média e alta), de modo a identificar as áreas de maior potencial.

\section{Resultados e Discussões}

As áreas potenciais definidas pela inferência espacial fuzzy foram avaliadas qualitativamente e quantitativamente, comparando-se os resultados com aqueles obtidos por Almeida-Filho (1995). Na análise qualitativa, observou-se como as 48 ocorrências minerais distribuíam-se em relação às diferentes classes de favorabilidade. A classe "alto potencial" encerrou 12 ocorrências minerais. Esse número salta para 27 (56\% das ocorrências), quando se agrega a ela a classe "médio potencial" (área conjunta =
$30,43 \mathrm{~km}^{2}$ ou $4,18 \%$ do maciço alcalino). Em contrapartida, os métodos empregados por Almeida-Filho (1995) mapearam 24 ocorrências na classe favorável indicada pelo método booleano (área $=$ $32,4 \mathrm{~km}^{2}$ ), enquanto o método "soma ponderada" mapeou 24 ocorrências minerais, juntando-se as classes alto e médio potencial (área $=30,64 \mathrm{~km}^{2}$ ). Esses dados indicam que o cenário fuzzy mostrou-se mais eficiente, mapeando 3 depósitos a mais, em áreas com superfícies equivalentes $\left(\cong 30 \mathrm{~km}^{2}\right)$.

$\mathrm{Na}$ análise quantitativa, foi utilizada a probabilidade condicional, para avaliar o caráter explicativo do cenário gerado em relação às verdades de campo (ocorrências minerais). Nessa análise, o parâmetro "grau de confiança" (Equação 6) mede a correlação entre as áreas potenciais e as ocorrências minerais. Se o modelo prospectivo adotado mostrarse coerente, espera-se alta correlação das ocorrências minerais com as áreas de favorabilidade mais alta. Valores (grau de confiança) expressarão a possibilidade de ocorrências minerais em determinada faixa de potencialidade.

Grau de confiança $=\frac{p(\text { depósito } \mid \text { fatia })}{p(d)}$

O grau de confiança, numa primeira análise, demonstrou que, tanto o cenário fuzzy, como os produzidos por Almei-

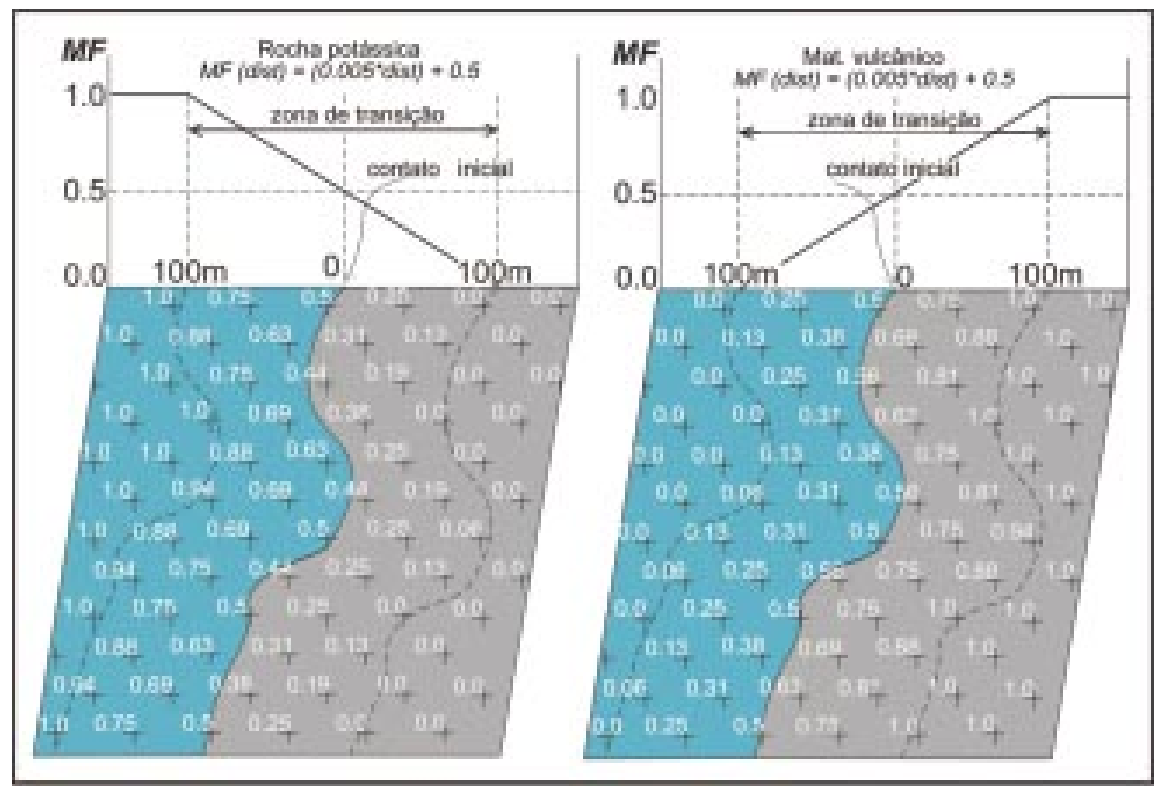

Figura 2 - Exemplos de membros fuzzy indicativos da zona de transição entre as unidades litológicas "rocha potássica" (azul) e material vulcânico (cinza). 
Tabela 1 - Sumário dos resultados obtidos pelos cenários de favorabilidade, através da análise qualitativa e quantitativa.

\begin{tabular}{c|c|c|c|c}
\hline \multicolumn{2}{c|}{} & Booleano* & Soma Ponderada & Fuzzy \\
\hline \multirow{4}{*}{ Classe Alto Potencial } & Área $\left(\mathrm{km}^{2}\right)$ & 32,37 & 6,48 & 6,14 \\
\cline { 2 - 5 } & Grau de confiança & 5,78 & 12,6 & 12,9 \\
\cline { 2 - 5 } & Depósitos & 24 & 12 & 12 \\
\hline \multirow{4}{*}{ Classe Médio Potencial } & Área $\left(\mathrm{km}^{2}\right)$ & - & 24,16 & 24,29 \\
\cline { 2 - 5 } & Grau de confiança & - & 4,97 & 5,7 \\
\cline { 2 - 5 } & Depósitos & - & 12 & 15 \\
\hline \multirow{2}{*}{ Alto + Médio potencial } & Área $\left(\mathrm{km}^{2}\right)$ & 32,37 & 30,64 & 30,43 \\
\cline { 2 - 5 } & Depósitos & 24 & 24 & 27 \\
\hline
\end{tabular}

* - resultados referentes à classe favorável.

da Filho (1995) geraram resultados coerentes, com valores baixos para as classes de baixa potencialidade (correlação negativa) e altos para as classes de alto potencial (correlação positiva). Por outro lado, os valores de grau de confiança das classes alto potencial $(12,9)$ e médio potencial $(5,7)$ do cenário fuzzy foram melhores quando comparados aos obtidos pelas respectivas classes do método soma ponderada, 12,6 e 4,97, respectivamente. A classe favorável do método booleano obteve 5,78 de grau de confiança. A Tabela 1 sumaria os resultados obtidos.

\section{Conclusões}

A abordagem da importação semântica (IS) permitiu, de modo prático, a incorporação da natureza imprecisa dos contatos (inferidos), o que representou um acréscimo de informação no modelamento. A consideração dessa nova variável em análises espaciais pode minimizar erros durante processos de tomada de decisão.

A possibilidade de representação dos dados em superfícies contínuas, pelo método $f u z z y$, permite maior flexibilidade na definição dos limiares de corte (tomada de decisão) e maior precisão no mapeamento das faixas de potencialidade.

Por essas características, pode-se afirmar que a teoria da lógica Fuzzy é bastante recomendável para estudos de fenômenos naturais.

\section{Referências Bibliográficas}

ALMEIDA-FILHO, R. Integração, manipulação e análise espacial de dados na pesquisa mineral através de modelos empíricos de prospecção: um exemplo no planalto de Poços de Caldas. Revista Brasileira de Geofísica, v.13, n.2, p.127-142, 1995.

ALMEIDA-FILHO, R., PARADELLA, W. R. Estudo do maciço alcalino de Poços de Caldas através da imagens Landsat com ênfase em mineralizações radioativas. São José dos Campos: Inpe-111-TPT/060, 1977. 130p.

ALTMAN, D. Fuzzy set theoretic approaches for handling imprecision in spatial analysis. International Journal of Geographical Information Systems, v.8, p.271-279, 1994.

BANAI, R. Fuzziness in Geographical Information System: contributions from analytic hierarchy process. International Journal of Geographical Information Systems, v.7, p.315-329, 1993.

BURROUGH, P. A. Fuzzy mathematical methods for soil survey and land evaluation. Journal of Soil Science, n.40, p.477-492, 1989.

BURROUGH, P. A., HEUVELINK, G. B. M. The sensitivity of Boolean and continuous (Fuzzy) logical modeling to uncertain data. In EGIS 92, 1992. Munich, Germany. Proceeding. Munich: EGIS 92, 1992. v.1, p.1032-1041.

BURRough, P. A., MCDONNELL, R. A. Principles of geographical information systems. Oxford: University Press, 1998. $333 \mathrm{p}$.

BUSHEE, J. A geochronological study of the alkalinemassif of Poços de Caldas, Brazil. Laboratório de Geocronologia da
Universidade de São Paulo. Relatório não publicado, 1970. 25p.

CÂMARA, G., SOUZA, R. C. M., FREITAS, U. M.,GARRIDO, J. C. P. SPRING. Integrating remote sensing and GIS with object-oriented data modelling. Computers and Graphics, v.15, p.13-22, 1996.

ELLERT, R. Contribuição à geologia do maciço alcalino de Poços de Caldas. São Paulo: Geologia, USP-FFCL, 1959, v.18, p.5-63.

FANG, J. H. Fuzzy logic \& geology. Geotimes: News and Trends in the Geoscience, v.42, p.23-26, 1997.

FRAENKEL, M. O., SANTOS, R. C., LOURENÇO, F. E. V. L., MUNIZ, W. S. Jazida de urânio no planalto de Poços de Caldas, Minas Gerais. In: Principais Depósitos Minerais do Brasil. DNPM, 1985. v.1, Cap.5, p.89-103.

NUCLEBRÁS - Empresas Nucleares Brasileiras. Mapa Geológico do Planalto de Poços de Caldas. 1975a. (Inédito).

NUCLEBRÁS - Empresas Nucleares Brasileiras. Mapa Radiométrico do Planalto de Poços de Caldas. 1975b. (Inédito).

OLIVEIRA, A. G. Mineralização de urânio e molibdênio no Planalto de Poços de Caldas. In: CONGRESSO BRASILEIRO DE GEOLOGIA, 28, 1974. Minas Gerais. Anais... Porto Alegre: SBG, 1974. v.1, p. 207-221.

SAATY, T. L. Multicriteria decision making the analytical hierarchy process. Pittsburgh: RWS Publications, 1992.

TOLBERT, J. The uraniferous zirconium deposits of the Poços de Caldas plateau, Brazil. U.S. Geological Survey Bulletin, 1966. 28p. (1185-C). 